\title{
Engineering Report on the Fission Gas Getter Concept
}

Fuel Cycle Research \& Development

Prepared for

U.S. Department of Energy FCR\&D Fuels Options Campaign

Lynne Ecker, Sanjit Ghose, Simerjeet Gill, BNL

Praveen K. Thallapally, Denis Strachan, PNNL

November 2012

FCRD-FUEL-2013-000059

PNNL-22138 


\section{DISCLAIMER}

This information was prepared as an account of work sponsored by an agency of the U.S. Government. Neither the U.S. Government nor any agency thereof, nor any of their employees, makes any warranty, expressed or implied, or assumes any legal liability or responsibility for the accuracy, completeness, or usefulness, of any information, apparatus, product, or process disclosed, or represents that its use would not infringe privately owned rights. References herein to any specific commercial product, process, or service by trade name, trade mark, manufacturer, or otherwise, does not necessarily constitute or imply its endorsement, recommendation, or favoring by the U.S. Government or any agency thereof. The views and opinions of authors expressed herein do not necessarily state or reflect those of the U.S. Government or any agency thereof. 


\section{TABLE OF CONTENTS}

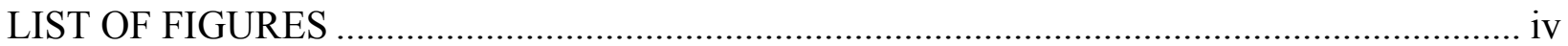

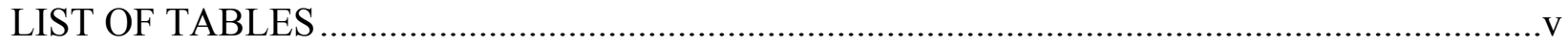

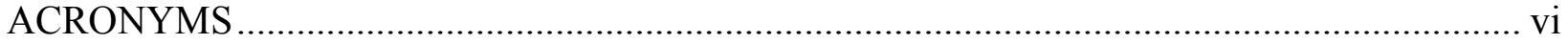

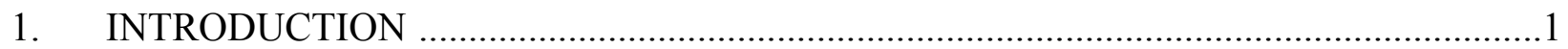

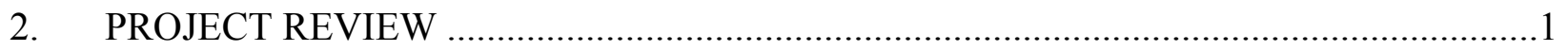

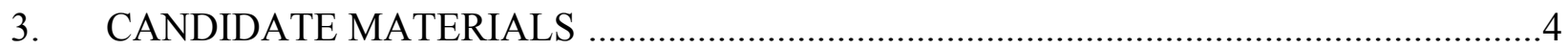

4. EXPERIMENTAL RESULTS FROM FEASIBILITY STUDIES AT THE

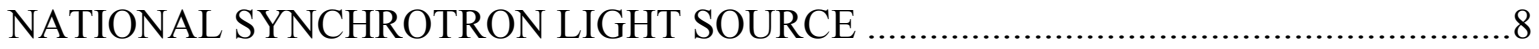

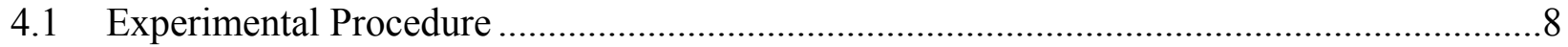

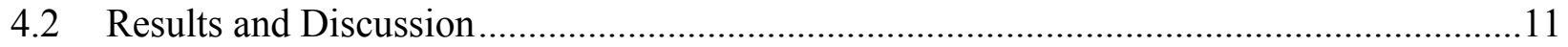

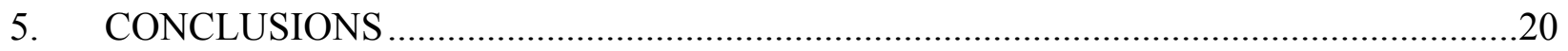

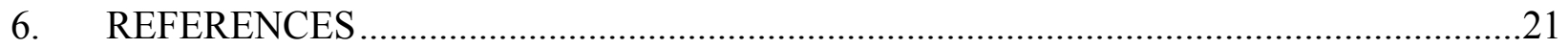

APPENDIX 1 EXPERIMENTAL TECHNIQUES …........................................................23 


\section{LIST OF FIGURES}

$\underline{\text { Figure }}$

$\underline{\text { Page }}$

1 Comparison between Langmuir adsorption isotherm and the BET adsorption isotherm

2 HRSEM analysis of as received (inactivated) Basolite

$3 \mathrm{Ni}$ /DOBDC crystal structure after removing solvent molecules...................................6

$4 \quad$ HRSEM micrograph of Carboxen ......................................................................... 6

$5 \quad$ HRSEM micrograph of Palladium Powder .............................................................. 7

$6 \quad$ HRSEM micrograph of Nickel Powder .....................................................................

7 Capillary tube with powder MOF sample at the beamline (orange) ...................................9

8 New automatic gas manifold and temperature controller designed and assembled at NSLS, BNL .................................................................................................. 10

9 Comparison between the Ni/DOBDC in the as received condition and after activation with subsequent exposure to humid air ................................................... 12

10 Comparison between the Ni/DOBDC after activation (a) and after exposure to

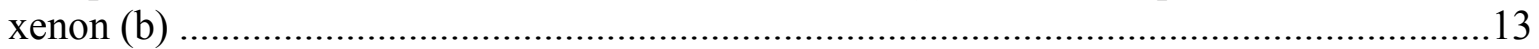

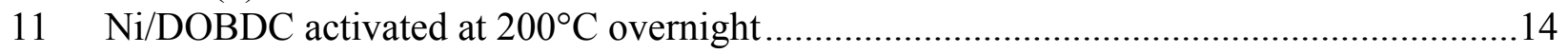

$12 \mathrm{XRD}$ data for the Ni/DOBDC MOF after activation and exposure to xenon gas ...............15

$13 \mathrm{XRD}$ data for Ni/DOBDC MOF after activation and exposure to xenon starting at $35^{\circ} \mathrm{C}$ for 60 minutes and increasing in temperature to $180^{\circ} \mathrm{C}$ in approximately

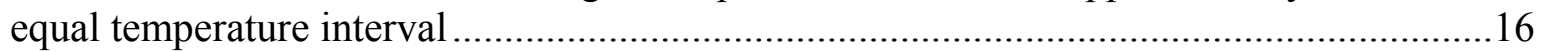

14 XRD data for Ni/DOBDC MOF after activation and exposure to krypton .......................18

$15 \mathrm{XRD}$ data for Basolite showing scans from $35-350^{\circ} \mathrm{C}$ in flowing xenon .........................19

A1 Schematic showing the solid sample surface during 1- degassing of the sample, 2-monolayer formation. 


\section{LIST OF TABLES}

$\underline{\text { Table }}$

$\underline{\text { Page }}$

1 Comparison of Surface Area and Binding Energy for Candidate Materials .4

2 Experimental Protocol Used at X17B3 $(80 \mathrm{keV})$ for Two Samples of Ni/DOBDC ............11

3 Experimental Protocol Used at X14A (16keV) for Ni/DOBDC Exposed to Xenon and Krypton 11

4 Experimental Protocol Used at X14A (16keV) for Basolite Exposed to Xenon .................11 


\section{ACRONYMS}

BET Brunauer, Emmett, and Teller

BNL Brookhaven National Laboratory

CMS Carbon Molecular Sieve

DOE U.S. Department of Energy

GISAXS Grazing Incidence Small Angle X-Ray Scattering

GIXD Grazing Incidence X-Ray Diffraction

HRXRD High-Resolution X-Ray Diffraction

LEED Low Energy Electron Diffraction

MOF Metal Organic Framework

NSLS National Synchrotron Light Source

PAX Photoemission of Adsorbed Xenon

PDF Pair Distribution Function

PNNL Pacific Northwest National Laboratory

TGA Thermogravimetric Analysis

XRD X-Ray Diffraction 


\section{INTRODUCTION}

In 2010, the Department of Energy (DOE) requested that a Brookhaven National Laboratory (BNL)-led team research the possibility of using a getter material to reduce the pressure in the plenum region of a light water reactor fuel rod. During the first two years of the project, several candidate materials were identified and tested using a variety of experimental techniques, most with xenon as a simulant for fission products. Earlier promising results for candidate getter materials were found to be incorrect, caused by poor experimental techniques. In May 2012, it had become clear that none of the initial materials had demonstrated the ability to adsorb xenon in the quantities and under the conditions needed. Moreover, the proposed corrective action plan could not meet the schedule needed by the project manager.

BNL initiated an internal project review which examined three questions:

1. Which materials, based on accepted materials models, might be capable of absorbing xenon?

2. Which experimental techniques are capable of not only detecting if xenon has been absorbed but also determine by what mechanism and the resulting molecular structure?

3. Are the results from the previous techniques useable now and in the future?

As part of the second question, the project review team evaluated the previous experimental technique to determine why incorrect results were reported in early 2012.

This engineering report is a summary of the current status of the project review, description of newly recommended experiments and results from feasibility studies at the National Synchrotron Light Source (NSLS).

\section{PROJECT REVIEW}

The first question addressed by the BNL review team was whether there existed a reasonable set of candidate materials that would adsorb xenon based on accepted materials models. The initial set of materials could include those which might not be stable under expected reactor conditions; in particular, those which have demonstrated success at lower temperatures.

As has been discussed in previous reports, there are two possible pathways for materials to adsorb xenon: physisorption and chemisorption. Physisorption is characteristic of weak bonding between the adsorbent and adsorbate, usually due to van der Walls forces. Chemisorption is characterized by stronger bonding, usually covalent, between the surface and adsorbent. In general, chemisorption is more appropriately described by the Langmuir theory, and the assumption that a monolayer of adsorbate molecules is deposited on the surface. Physisorption is better characterized by Brunauer, Emmett and Teller (BET) theory, which considers the formation of multilayers of absorbed molecules on a surface. Adsorption is usually described through isotherms, which are the amount of adsorbate on the adsorbent as a function of its pressure at a constant temperature [1]. The difference in adsorption isotherms predicted by each theory is shown in Figure 1. 


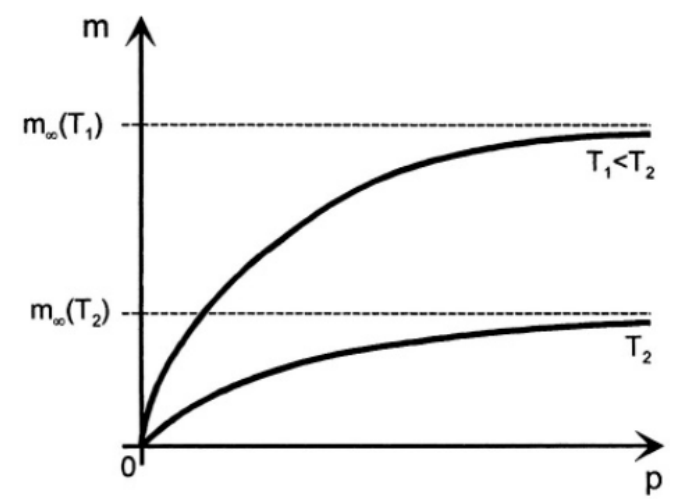

(a) The classical Langmuir adsorption isotherm sketched for two different temperatures $\left(T_{1}<T_{2}\right)$. The limiting loadings increase with decreasing temperature, i. e. $\mathbf{m}_{\infty}\left(\mathbf{T}_{2}\right)<\mathbf{m}_{\infty}\left(\mathbf{T}_{1}\right)$

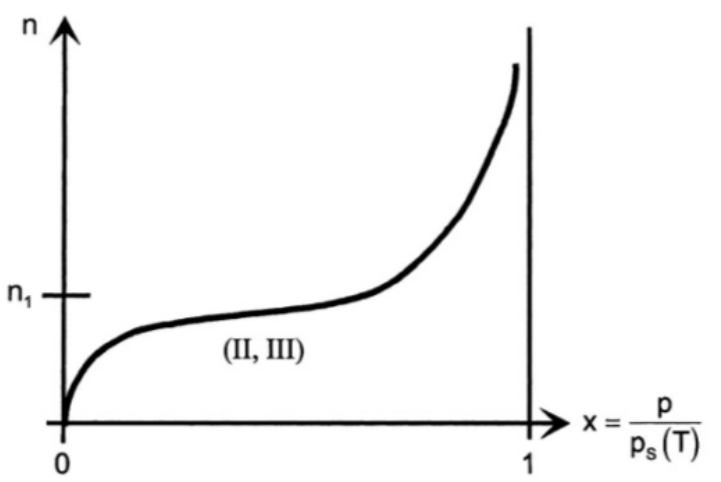

(b) The BET adsorption isotherm showing an inflection point at the sorptive gas saturation pressure $p=p_{s}(T)$ and a singularity $(n \rightarrow \infty)$ indicating pore condensation and the appearance of a bulk liquid phase.

Figure 1. Comparison between Langmuir adsorption isotherm and the BET adsorption isotherm. ( $\mathrm{m}$ is mass of the adsorbate, $\mathrm{T}$ is temperature, $\mathrm{P}$ is pressure and $\mathrm{n}$ is the amount of gas adsorbed on a sorbent) [Figure taken from Reference 2].

The internal BNL review team consisted of experts on adsorption for gettering in vacuum science, hydrogen storage, chemistry and condensed matter physics. In addressing the first question (candidate getter materials), all the experts consulted for this review were highly skeptical that it would be possible to getter the fission gas using adsorption on the surface of a bulk material. This is due the fact that the bond between the noble gases and surfaces will not be strong enough to maintain the adsorption at reactor temperatures. For example, the xenon desorption energy from the tungsten 111 surface is $8 \mathrm{kcal} / \mathrm{mol}$ [3]. From this, the residence time for xenon on this surface is estimated to be on the order of $10^{-10}$ seconds. In general, the desorption energies for xenon are reported to be on the order of 3-6 Kcal/mol and the typical desorption energies for chemisorptions are on the order of $20 \mathrm{kcal} / \mathrm{mol}$. This indicates that the surfaces of these materials are not acting as getters for noble gases. None of the experts, including a specialist in electron interactions, indicated that using nanopowders would fundamentally change this physics. In fact, there are no getter materials for xenon gas. One textbook states that "noble gases are not sorbed by getter materials." It is known that one can chemosorb xenon on palladium in the presence of fluorine at moderate temperatures; however, this is a catalytic reaction and requires fluorine to work [4-6].

The experts then identified the performance needed for a material to adsorb a gas and hold it for a reasonable time (a week to a year) at room temperature. This requires an energy of desorption of approximately $25-30 \mathrm{Kcal} / \mathrm{mol}$ [4], and desorption energies in this range are required before it is possible to consider a material a getter for fission gases. It was generally agreed that the best possibility to lower the fission gas pressure in the plenum might be a material that confines the fission gas in internal porosity. It may be possible to "tune" the size of the internal porosity in known materials to retain xenon and krypton. Materials that may be capable of this include Metal Organic Frameworks (MOFs), intercalated clays, and carbon nanostructures. However, it must be acknowledged that this is high-risk research and none of the experts indicated that success was assured [6]. 
It should be noted that the He pressure in a boiling water reactor fuel assembly is on the order of $3 \mathrm{~atm}$ at a temperature of $600^{\circ} \mathrm{K}$. Therefore, the mean free path for xenon is on the order of microns. Diffusion alone is unlikely to occur quickly enough to transport the xenon to a remote getter material [4]. This is a concern regardless of the material selection and will be addressed in the next progress report.

In addressing the second question, the experts concluded that standard techniques be considered for measuring surface area, pore volume and adsorption of noble gases on candidate getter materials. These are BET measurements and direct gas adsorption measurements and are discussed in more fully in Appendix 1. The previous experiments at BNL were not able to characterize whether the xenon was present on the surface of the candidate getter materials. Powder X-ray Diffraction and Extended Absorption Fine Structure measurements are not surface sensitive in the configurations that were used for the studies. In addition, it is not obvious that atmospheric gases would not have displaced xenon adsorbed on a surface in the Thermogravimetric analysis (TGA) when it was removed for characterization. The project review indicates that two of the more common techniques for observing xenon on a surface are Low Energy Electron Diffraction (LEED) and Photoemission of Adsorbed Xenon (PAX) (more fully described in Appendix 1).

In addressing question 3, the experts also generally agreed that the TGA experiments performed at BNL were not the optimal experiment to measure the adsorption of noble gases. This is primarily due to the absence of a residual gas analyzer so the species being desorbed is unknown. Difficulties removing the oxygen from this system also led to the misinterpretation of weight gain of nickel powder as xenon adsorption as opposed to the oxidation of nickel. The TGA experiments lacked a consistent, well-documented experimental protocol. These difficulties make conclusions based on this data subject to reevaluation. In particular, carbon-based materials were removed from consideration because they decompose at reactor temperatures, and metal powders were selected based largely on the weight gain in the TGA due to oxidation and the hope that the materials would exhibit chemisorption if nanosized particles were used. The engineering project review reconsidered the material selection and specifically asked the BNL experts to recommend materials [5].

The previous investigator concluded from his literature review and the TGA experiments that the reason that the xenon was not chemisorbed to the surface of the Nickel and palladium powder was that the surface of the powder was covered by a monolayer of oxygen. He designed an experiment using an autoclave to heat the powders in a hydrogen atmosphere to reduce any oxygen on the surface. The protocol then required heating and pressurizing the samples with xenon. The experts generally concurred that this experiment would still be inconclusive because of the possibility of forming a hydride surface layer and because no method was identified that could definitively determine if xenon was adsorbed on the surface. It was recommended that if this experimental effort continued a very sensitive method of directly measuring pressure be used to monitor the experiment and a residual gas analyzer be used to measure the desorbed gases. BNL is still evaluating whether to pursue these experiments and, if so, to determine an experimental test protocol [5]. 


\section{CANDIDATE MATERIALS}

As a result of the project review, both materials used previously in the TGA studies and new candidate materials (Table 1) are being considered to getter fission gases. This section briefly describes the materials currently being considered and, where appropriate, explains the mechanism that may offer the potential to adsorb fission gases and modifications to the material that could increase the chances of retaining fission gasses at reactor temperatures and pressures.

Based on the project review and the fact that gettering noble gases are such a challenging goal, materials are selected solely on the basis of their potential to retain fission gases at moderate temperatures. If such materials can be identified and the mechanisms that allows this functionality described, it may be possible to create new, high-temperature, radiation-resistant materials capable of retaining fission gases.

Table 1. Comparison of Surface Area and Binding Energy for Candidate Materials

\begin{tabular}{||c|c|c|c|c|}
\hline Material & $\begin{array}{c}\text { Surface Area } \\
\mathbf{m} \mathbf{2} / \mathbf{g}\end{array}$ & Reference & $\begin{array}{c}\text { Binding energy } \\
\mathbf{k c a l} / \mathbf{m o l}\end{array}$ & Reference \\
\hline \hline $\begin{array}{c}\text { Cu MOF } \\
\text { Basolite C 300 }\end{array}$ & $1500-2100$ & {$[26]$} & 5.0 & {$[25]$} \\
\hline $\begin{array}{c}\text { Ni/DOBDC } \\
\text { MOF }\end{array}$ & 950 & {$[9]$} & 5.3 & {$[9]$} \\
\hline $\begin{array}{c}\text { Microporous Carbon } \\
\text { Carboxen/ Carbosieve }\end{array}$ & 400 & {$[26]$} & 5.3 & {$[15]$} \\
\hline $\begin{array}{c}\text { Pd } \\
99.9 \% \text { purity }\end{array}$ & & & $\begin{array}{c}8.3 \text { for }(111) \\
\text { surface }\end{array}$ & {$[18]$} \\
\hline SWCNT & 330 & {$[20]$} & 6.4 & {$[21]$} \\
\hline $\begin{array}{c}\text { Organo-Clay } \\
\text { TEQ-hectorite }\end{array}$ & & 3.3 & {$[20]$} \\
\hline
\end{tabular}

In some cases, the surface morphology of the candidate materials was examined using Analytical High Resolution SEM JEOL 7600F. Samples were prepared by spraying a small amount of the sample powders onto a carbon tape fitted on an aluminum sample holder. Once in the sample chamber, the specimen was allowed to outgas and equilibrate under vacuum and electron-beam bombardment, at low magnification, for 5-10 minutes. For analysis of surface morphology, low accelerating voltage of $2 \mathrm{kV}$ was selected to minimize both the effects of charging and sample damage.

The candidate materials currently being considered are:

1. Metal Organic Frameworks. MOFs are hybrid lattices of organic electron donors and metal cations and are unique in their flexibility to tune the pore size and chemical properties of the pores [7]. MOFs are being considered for separation of fission gases (xenon from krypton) at low temperature [8-10]. The project review indicated that the most promising materials to getter the fission gas may confine the gas molecules in internal porosity; therefore, MOFs are likely candidate materials. Experiments are currently being performed at the NSLS to provide information on the structural behavior of MOFs and xenon or krypton interacting. Two candidate MOF materials that are currently being used for experiments at the NSLS are: 
- Basolite HKUST-1 (BasoliteÒ C-300 from Sigma-Aldrich) is a Cu-based MOF that shows chemisorptions (Langmuir isotherms) with $\mathrm{CO}_{2}, \mathrm{CH}_{4}$ and $\mathrm{N}_{2}$. It was also reported to be highly crystalline, with grain size on the order of approximately 5-10 micron $[11,12]$. HRSEM micrographs on the material tested at BNL purchased from SigmaAldrich are shown in Figure 2 and display faceted crystalline particles (Figure 2A) with micron-sized grains. The bright areas in the center of some of the facets are due to charging of the samples in the HRSEM. Although inclusive, TGA experiments at BNL did suggest that Basolite was adsorbing xenon gas.

- $\mathrm{Ni} / \mathrm{DOBDC}$ has a honeycomb network structure shown in the open literature (Figure 3) and is also known to adsorb xenon and krypton [9, 10, 13, 14]. BNL received samples of $\mathrm{Ni} / \mathrm{DOBDC}$ from Pacific Northwest National Laboratory (PNNL) and is currently in the process of establishing a nondisclosure agreement with PNNL that is needed before further characterizing the samples.
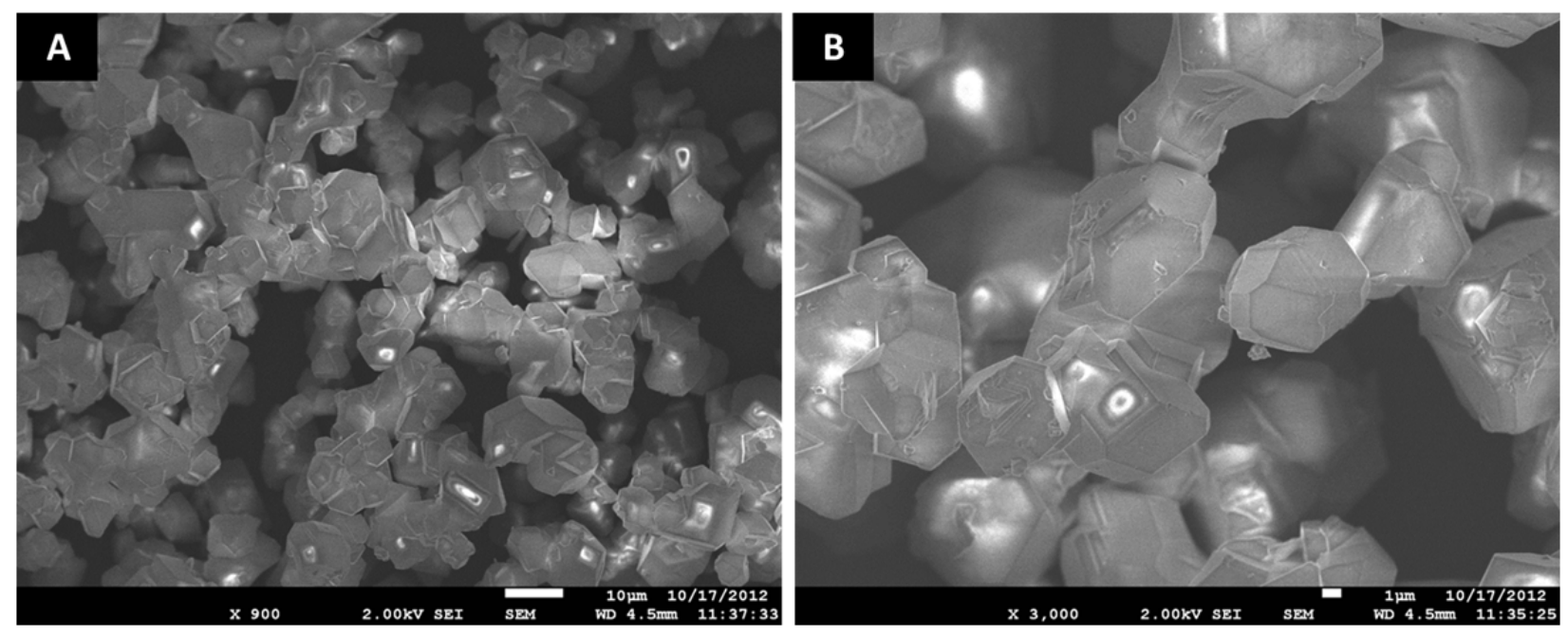

Figure 2. HRSEM analysis of as received (inactivated) Basolite. 


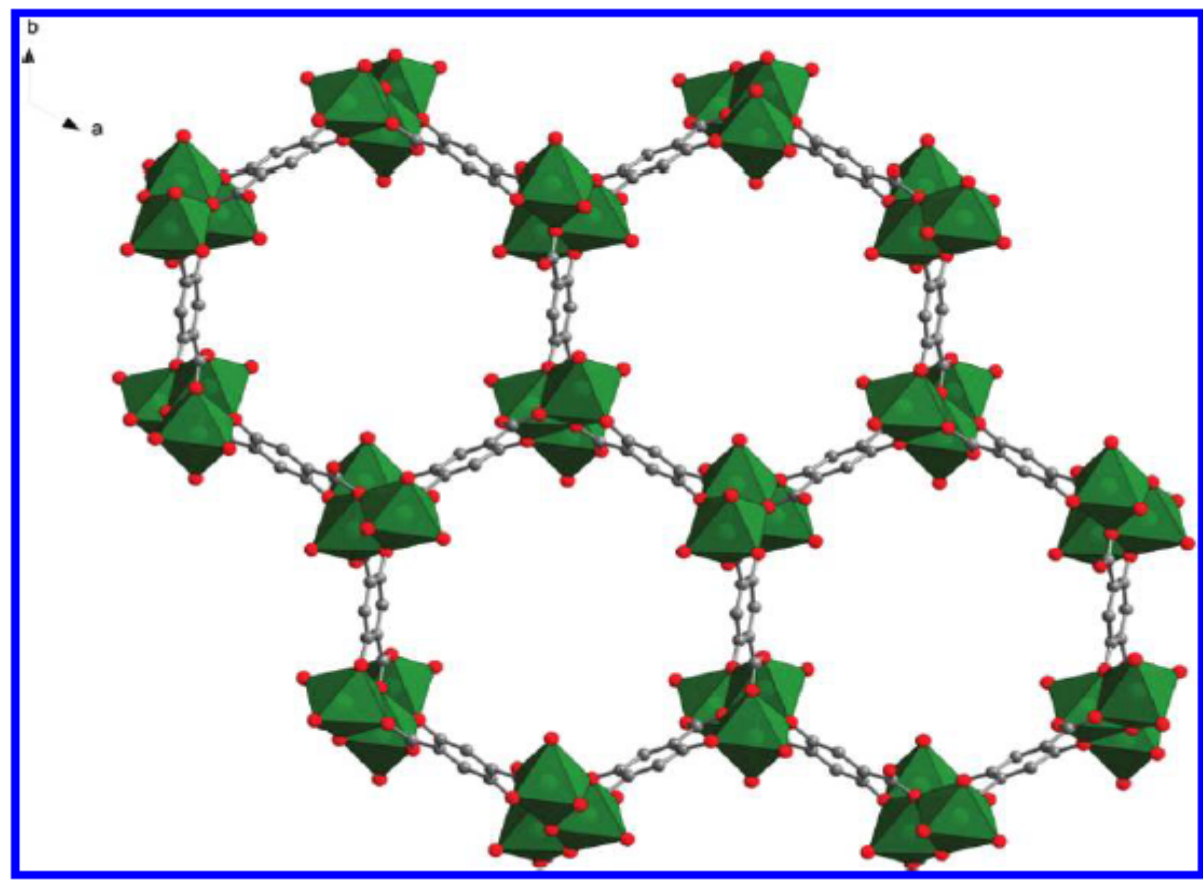

Figure 3. Ni/DOBDC crystal structure after removing solvent molecules. Ni atoms: green polyhedra; $O$ atoms: red spheres; $C$ atoms: gray spheres [Taken from Reference 13].

2. Carbon Molecular Sieve (CMS) Adsorbents. Activated carbon (carbon processed to contain small pores) is commonly used in filtering to remove impurities by adsorption. Studies suggest that it is almost as efficient in adsorbing xenon and krypton as MOF materials [9] and that the xenon may be stabilized by forming clusters in the micropores [15]. SigmaAldrich offers two types of CMS adsorbents: (1) Carboxen adsorbents, which have throughpore structures, resulting in efficient adsorption/desorption characteristics and (2) Carbosieve adsorbents, which have closed-pore structures, resulting in strong adsorption characteristics [26]. The Carboxen tested in the TGA at BNL did appear to increase in mass in the presence of xenon. A micrograph of the material tested is shown in Figure 4 and demonstrates that the material provided consists of spheres with diameters on the order of hundreds of microns.
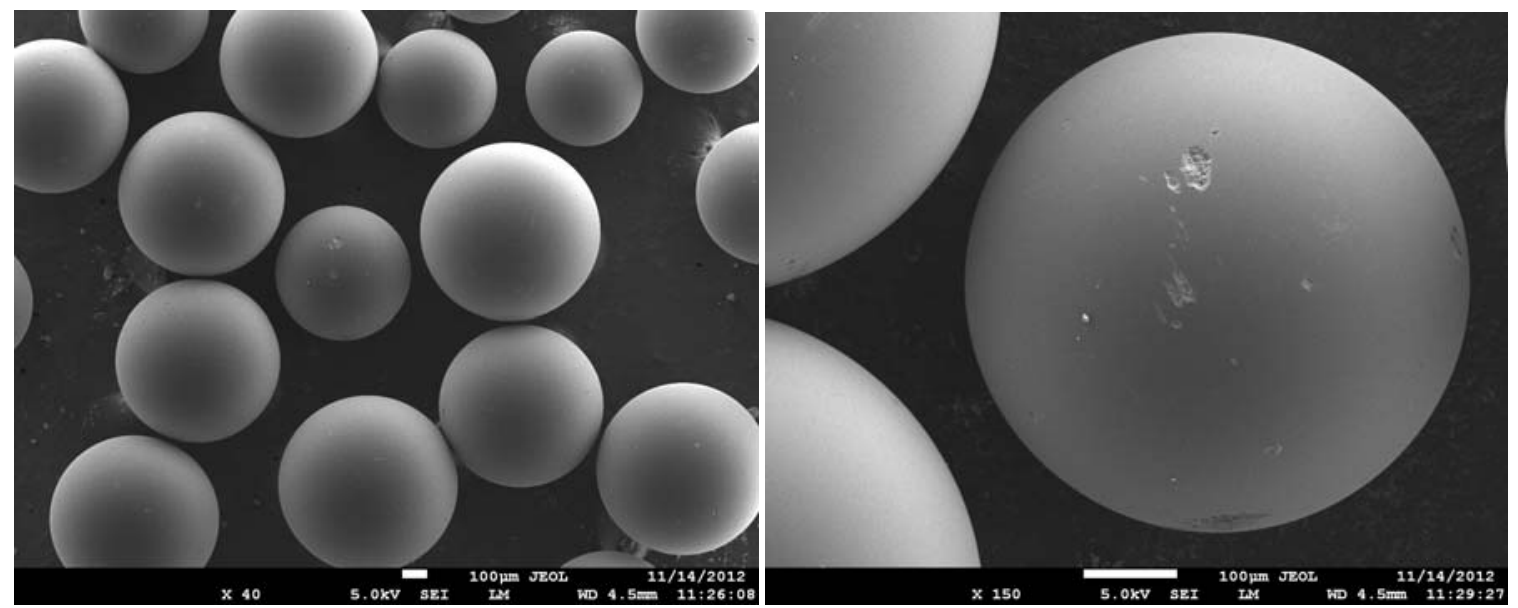

Figure 4. HRSEM Micrograph of Carboxen. 
3. Palladium and Nickel Powder. The literature does support xenon adsorption on palladium and nickel powders [16-19]. It has been suggested that the xenon will polarize as it approaches the palladium surface (London dispersion forces). Palladium powder is still being considered as a candidate getter material; however, the HRSEM micrographs in Figures 5 and 6 indicate that the palladium and nickel powders already obtained demonstrate highly porous structure with interconnected porosity (Figure 5A) and individual particle size in the nanometer regime (Figure 5B). It is doubtful, based on information provided during the project review and the micrographs, that purchasing or fabricating palladium nanopowder will greatly increase the surface area or change the character of the bonding of xenon with the surface. Experiments with the materials shown in Figures 5 and 6 will be evaluated to determine if additional palladium and nickel powders will be procured.

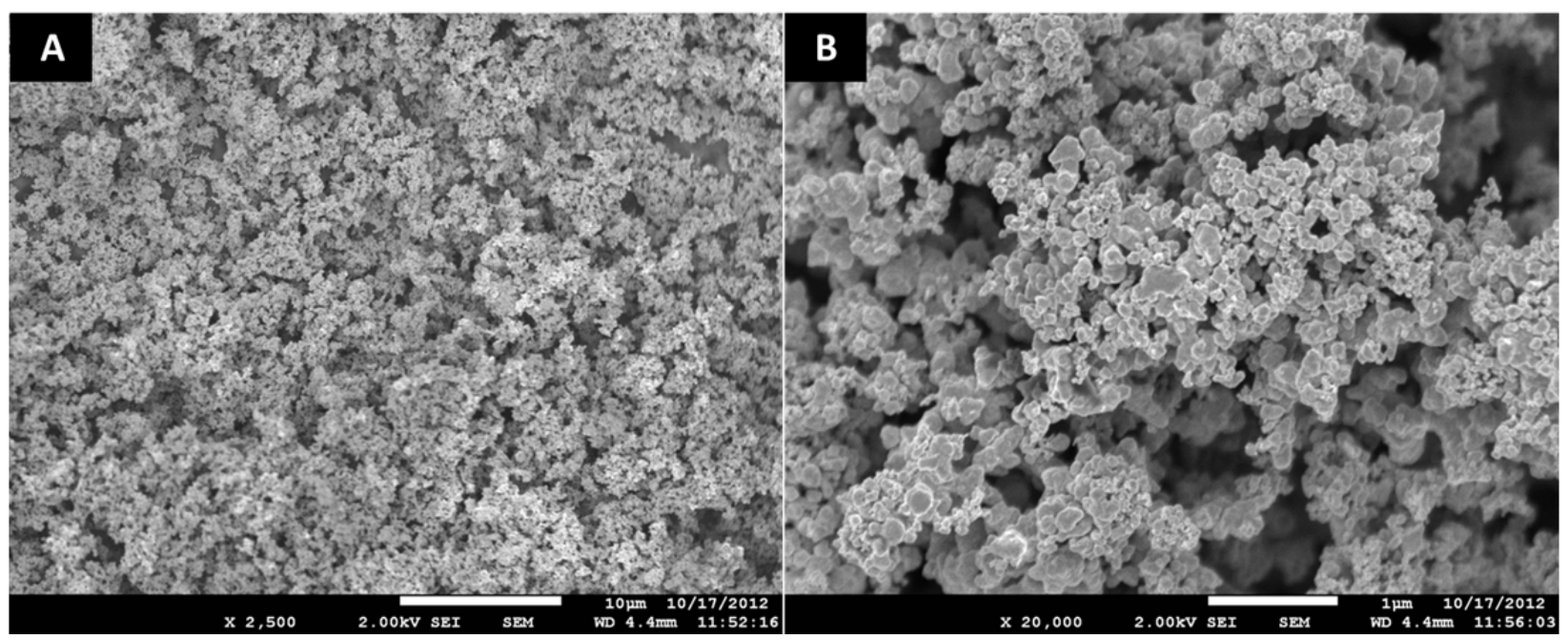

Figure 5. HRSEM Micrograph of Palladium Powder.
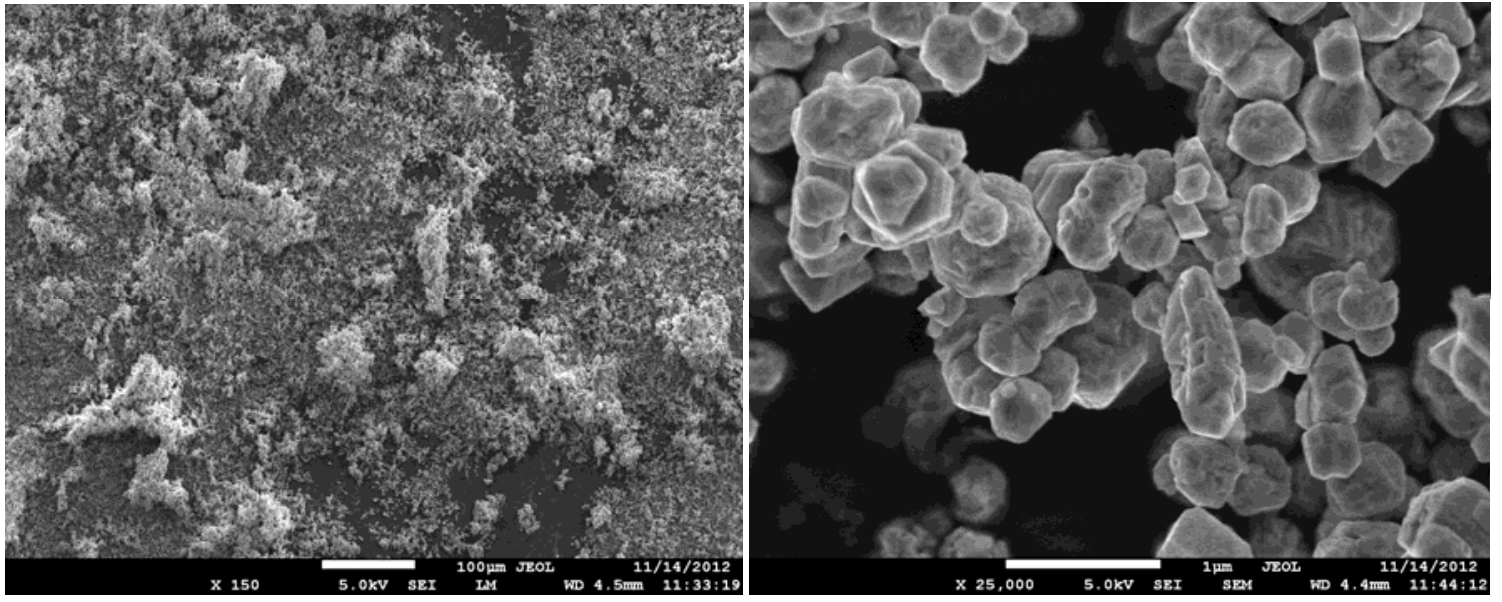

Figure 6. HRSEM Micrograph of Nickel Powder.

4. Intercalation Compounds. The project review recommended consideration of nanolayered materials with porosity between the layers (intercalation). A brief literature search did suggest an organo-clay (hectorite pillared by tetraethyl-ammonium ions, TEA-hectorite) that adsorbed xenon (see Table 1) [20]. It may be possible to adjust the interlayer distance in these materials to more strongly adsorb xenon. 
5. Other Materials. Other candidate getter materials that will be reconsidered include singleand double-walled carbon nanotubes and zeolites. Carbon nanotubes demonstrate the adsorption of noble gases into porosity within the tubes [21, 22]. Zeolites have tunable pore sizes and may be capable of confining xenon to the pores [23, 24].

\section{EXPERIMENTAL RESULTS FROM FEASIBILITY STUDIES AT THE NATIONAL SYNCHROTRON LIGHT SOURCE}

In situ experiments were performed at the NSLS of BNL to determine the feasibility of identifying the structural changes in MOF materials from the adsorption of fission gases. Often, it is difficult to predict the structure of physisorbed inert gases like xenon and krypton into a bulk matrix from powder diffraction. However, powder X-Ray Diffraction (XRD) measurement provides structural information on the crystal structure of the bulk material and the corresponding structural changes that occur due to xenon and krypton. This experiment used powder XRD to observe the crystal structure of MOF materials as received, after activation and after exposure to xenon and krypton and further structural changes with isothermal studies. It is noted that changes in the bulk crystal structure for many getter materials are not expected and that these experiments are useful for the MOF materials and possibly other materials that may adsorb fission gases in internal porosity if that adsorption results in bond stretching or in ordering of the fission gases in the lattice. These techniques are not expected to be useful in evaluating the metal powders because the bulk crystal structure will not change due to gas adsorption on the surface.

\subsection{Experimental Procedure}

The MOF material samples are hydrophilic and will adsorb water on the surface in room temperature air. Therefore, the samples need to be activated by heating in helium to drive off moisture adsorbed on the surface. The nominal experimental procedure is to heat the MOF in flowing helium in an environmental chamber at the beamline while acquiring XRD data. Next, the MOF is exposed to either flowing xenon or krypton also while monitoring the crystal structure with XRD. The environmental chamber is shown in Figure 7, and a new automatic gas manifold and temperature controller is shown in Figure 8. Experiments were performed at two beamlines at the NSLS. X17B3 was used to obtain data at $80 \mathrm{keV}$ that has the potential to be used for Pair Distribution Function (PDF) analysis, and X14A was used to obtain HighResolution X-Ray Diffraction (HRXRD) data at $16 \mathrm{keV}$. The specific experimental protocol for each sample and beamline is given in Tables 2-4. 


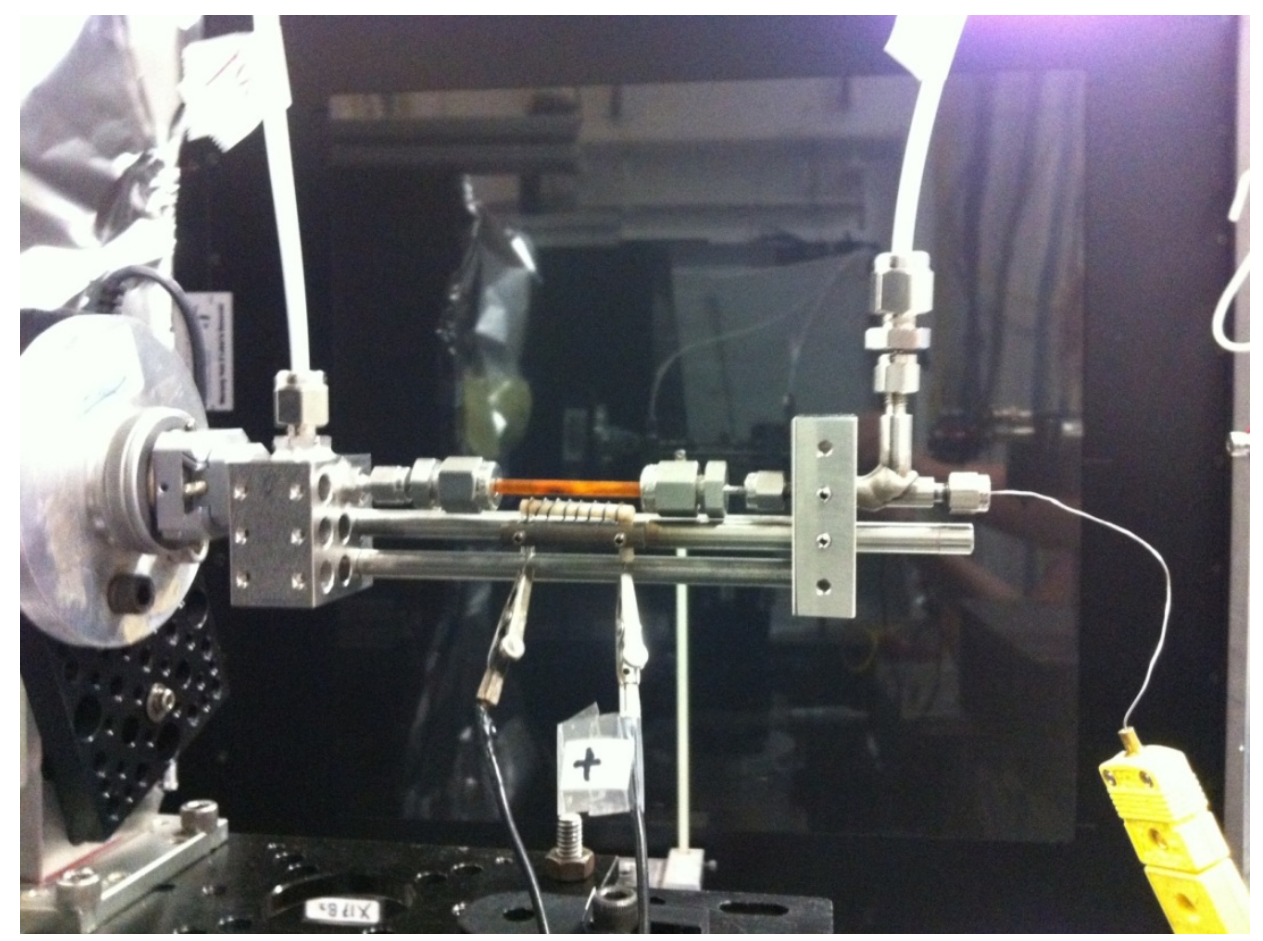

Figure 7. Capillary tube with powder MOF sample at the beamline (orange).

Heating coils are positioned beneath the sample. Gas flow is through the plastic tubing into and out of the capillary tube. Heating coils and temperature sensors are shown in the picture. The beam intersects the sample into the page. 


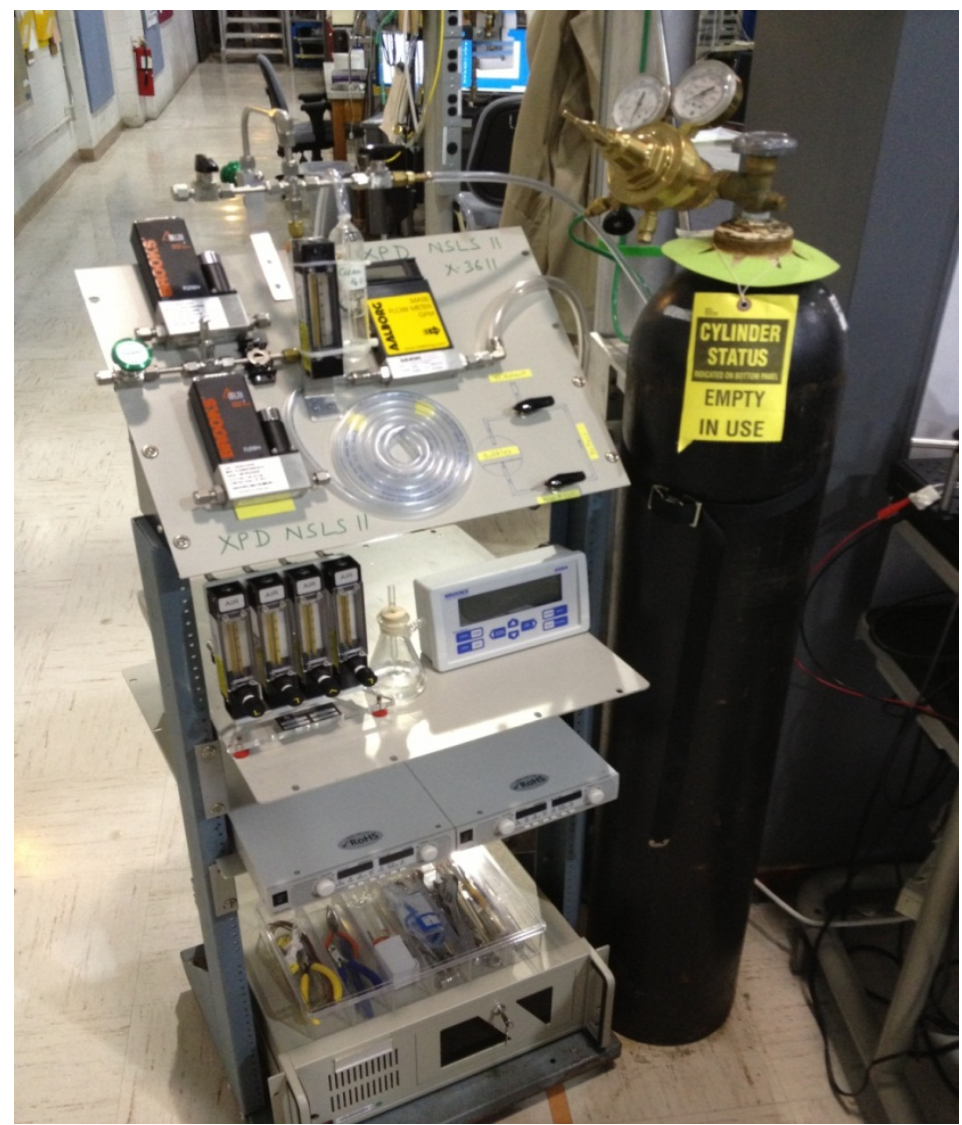

Figure 8. New automatic gas manifold and temperature controller designed and assembled at NSLS, BNL.

This compact and portable system is compatible with any beamline for in situ experiments with temperature and gas flow control. The picture shows helium gas cylinder, regulator, automatic gas flow meters, heater power supply and temperature controller. The first high resolution and fast XRD experiment on the Ni-DOBDC and Basolite MOFs were performed with xenon \& krypton adsorption. 
Table 2. Experimental Protocol Used at X17B3 (80keV) for Two Samples of Ni/DOBDC.

\begin{tabular}{|c|c|c|c|}
\hline Ni/DOBDC & Time & Temperature & Gas Flow \\
\hline \hline Sample 1 & 12 hours & $200^{\circ} \mathrm{C}$ & $\mathrm{He}$ \\
\hline & 12 hours & $\sim 30^{\circ} \mathrm{C}$ & Humid Air \\
\hline Sample 2 & 12 hours & $200^{\circ} \mathrm{C}$ & $\mathrm{He}$ \\
\hline & 0.5 hours & $\sim 30^{\circ} \mathrm{C}$ & $\mathrm{Xe}(\geq 1$ bar $)$ \\
\hline & 10 hours & $\sim 30^{\circ} \mathrm{C}$ & $\mathrm{Xe}(\geq 1$ bar $)$ \\
\hline
\end{tabular}

Table 3. Experimental Protocol Used at X14A (16 keV) for Ni/DOBDC Exposed to Xenon and Krypton.

\begin{tabular}{|c|c|c|c|}
\hline \hline Ni/DOBDC & Time & Temperature & Gas Flow \\
\hline \hline Sample 1 & 8 hours & $140^{\circ} \mathrm{C}$ & $\mathrm{H} 3$ \\
\hline & 30 minutes & $140^{\circ} \mathrm{C}$ & $\mathrm{Xe}(\geq 1 \mathrm{bar})$ \\
\hline & 60 minutes & $35^{\circ} \mathrm{C}$ & $\mathrm{Xe}(\geq 1 \mathrm{bar})$ \\
\hline & 2 hours & $35-250^{\circ} \mathrm{C}$ & No gas \\
\hline Sample 2 & 8 hours & $140^{\circ} \mathrm{C}$ & $\mathrm{H} 3$ \\
\hline & 30 minutes & $140^{\circ} \mathrm{C}$ & $\mathrm{Kr}(\geq 1$ bar $)$ \\
\hline & 60 minutes & $35^{\circ} \mathrm{C}$ & $\mathrm{Kr}(\geq 1$ bar $)$ \\
\hline & 2 hours & $35-350^{\circ} \mathrm{C}$ & No gas \\
\hline
\end{tabular}

Table 4. Experimental Protocol Used at X14A (16 keV) for Basolite Exposed to Xenon.

\begin{tabular}{|c|c|c|c|}
\hline Basolite & Time & Temperature & Gas Flow \\
\hline \hline Sample 1 & 8 hours & $140^{\circ} \mathrm{C}$ & $\mathrm{He}$ \\
\hline & 30 minutes & $140^{\circ} \mathrm{C}$ & $\mathrm{Xe}(\geq 1 \mathrm{bar})$ \\
\hline & 60 minutes & $35^{\circ} \mathrm{C}$ & $\mathrm{Xe}(\geq 1 \mathrm{bar})$ \\
\hline & 2 hours & $35-250^{\circ} \mathrm{C}$ & No gas \\
\hline
\end{tabular}

\subsection{Results and Discussion}

Results for the XRD data from $80 \mathrm{keV}$ for the Ni/DOBDC are shown for the samples as-received (exposed to air), after activation and after exposure to humid air and xenon (Figures 9 and 10). The graphs are the measure of the intensity of the diffracted beam (in counts) versus Q (1/nm). $\mathrm{Q}$ is the magnitude of the scattering vector. For elastic scattering, this is $4 \pi \sin (\Theta) / \lambda$ with $2 \Theta$ equal to the scattering angle and $\lambda$ equal to the wavelength of the beam. Qualitative analysis was done on the data for observing any structural modification. Comparison of Figures $9 \mathrm{a}$ and $9 \mathrm{~b}$ reveals very little difference in the spectra between as-received $\mathrm{Ni} / \mathrm{DOBDC}$ and the Ni/DOBDC after activation and exposure to humid air. By contrast, comparison between Figure 10a and Figures 9a and 9b shows a difference in the intensity at a $\mathrm{Q}$ of approximately $13-14 \mathrm{~nm}^{-1}$. This may be due to the as-received samples adsorbing water from the room temperature air. Therefore, it is possible to observe a difference in the spectra for the Ni/DOBDC MOF before and after activation, and this difference is attributed to slight changes in the MOF due to the adsorption of moisture. 
Comparison between the intensity of diffraction data from the activated Ni/DOBDC (Figure 10a) and the Ni/DOBDC (Figure 10b) after exposure to xenon show a clear difference with peak splitting at a $Q$ of approximately $10 \mathrm{~nm}^{-1}$ and new peaks appearing at a $\mathrm{Q}$ of $18-20 \mathrm{~nm}^{-1}$. These results indicate that the in situ observation of an interaction between the Ni/DOBDC and xenon is possible. However, it is difficult to interpret the exact changes in structure that are being observed. Two possibilities are that the xenon is stretching bonds or otherwise distorting the structure of the MOF when it is adsorbed or that the xenon-xenon distances are ordered by their incorporation in the MOF lattice. PDF analysis from the high Q data would reveal the Xe-Xe distances. Unfortunately, this preliminary data was too noisy to calculate an adequate PDF to determine which phenomenon is occurring. Future plans include repeating the measurements at high Q using high energy x-rays and performing the PDF analysis.

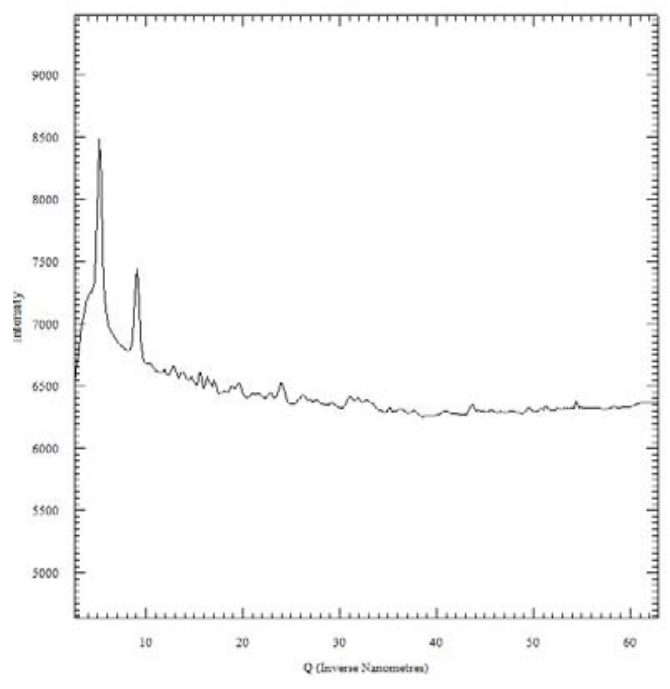

(a) Sample exposed to air (before activation)

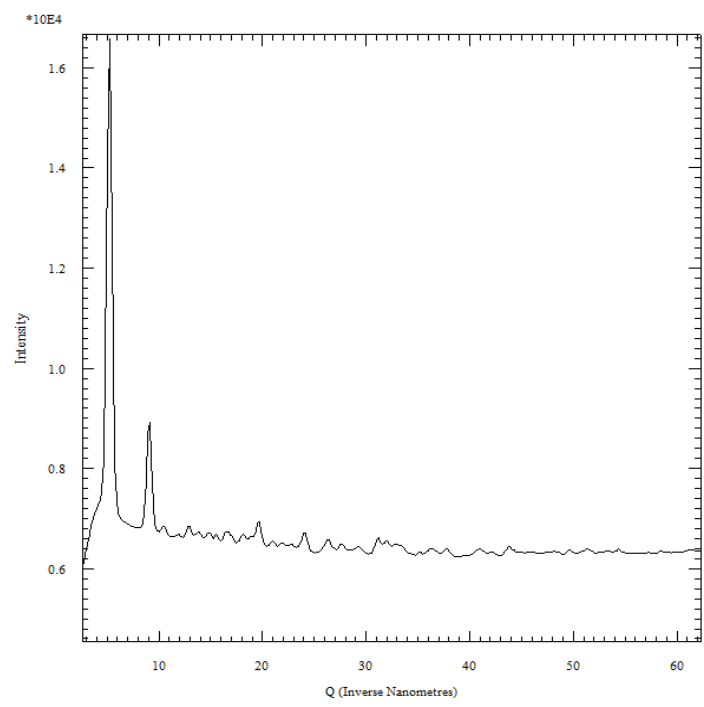

(b) Sample after activation and exposure to humid air

Figure 9. Comparison between the Ni/DOBDC in the as received condition and after activation with subsequent exposure to humid air.

The data shown are the XRD part of the total X-ray scattering data taken for only $4 \mathrm{~s}$ at $80 \mathrm{keV}$ using a 2D detector. 


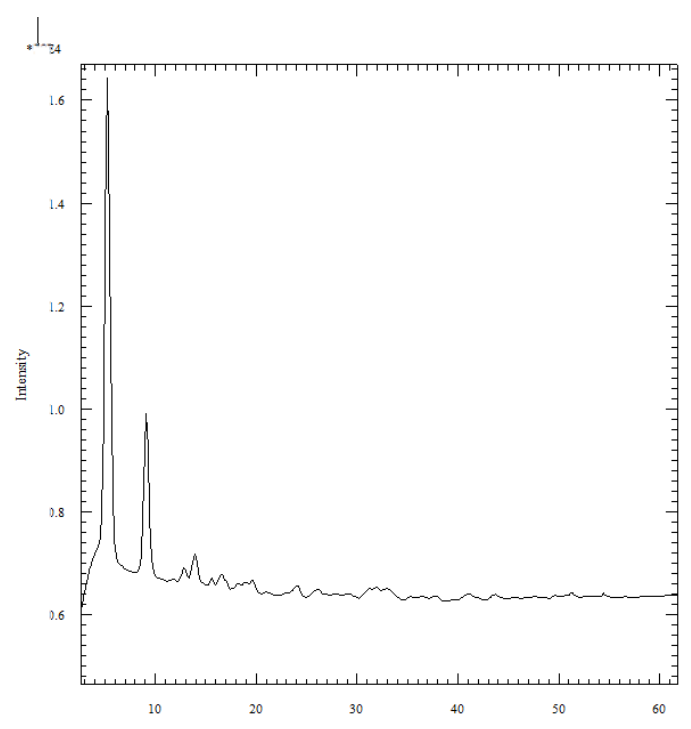

(a) After Activation in He. Sample at $200{ }^{\circ} \mathrm{C}$

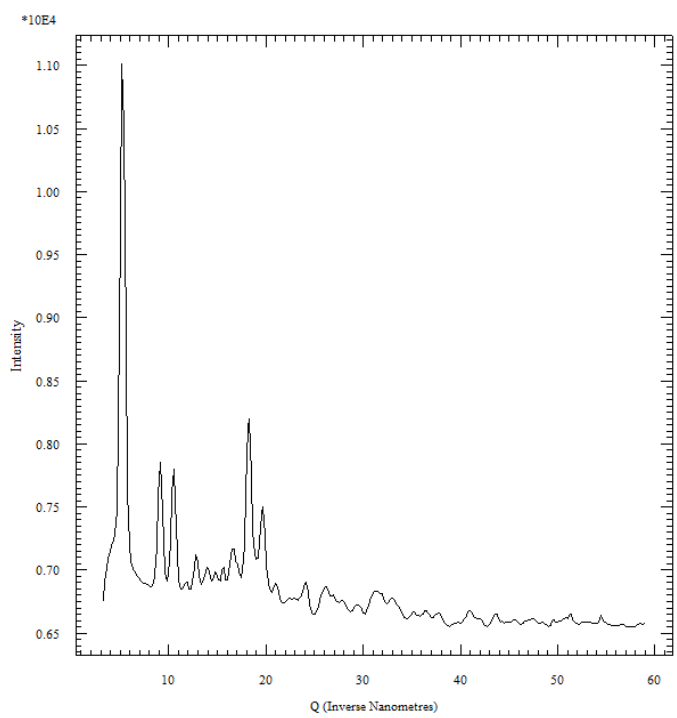

(b) Sample after30 minutes exposure to Xe at room temperature

Figure 10. Comparison between the Ni/DOBDC after activation (a) and after exposure to xenon (b).

The peak near $10 \mathrm{~nm}^{-1}$ is split and several new peaks can be seen, demonstrating that the exposure to xenon changes the MOF XRD spectra, probably the result of xenon absorption. 
HRXRD data was obtained at $16 \mathrm{keV}$ on the $\mathrm{Ni} / \mathrm{DOBDC}$ and Basolite to determine if structural changes that could be resolved were occurring in the MOF due to the exposure to xenon. Results from the first sample of Ni/DOBDC are shown in Figure 11. The sample was activated according to the experimental protocol used in the previous studies: 12 hours in flowing helium at $200^{\circ} \mathrm{C}$. However, in this case, the quality of the data deteriorated over time. The early spectra show diffraction peaks, the intermediate spectra show broadening of these peaks due to the material grain size decreasing, and the later spectra show smooth curves without peaks indicating amorphous material at about $180^{\circ} \mathrm{C}$. It is not known why the material remained highly crystalline to $200^{\circ} \mathrm{C}$ temperatures at beamline $\mathrm{X} 17 \mathrm{~B} 3$, but showed deterioration in the XRD spectrum quality at X14A. Previous studies of Ni/DOBDC indicate that it retains its structure to at least $250^{\circ} \mathrm{C}$ [13]. A new activation protocol was established to heat the material to $140^{\circ} \mathrm{C}$ in flowing helium for 12 hours. No deterioration in the XRD spectra was observed at these temperatures.

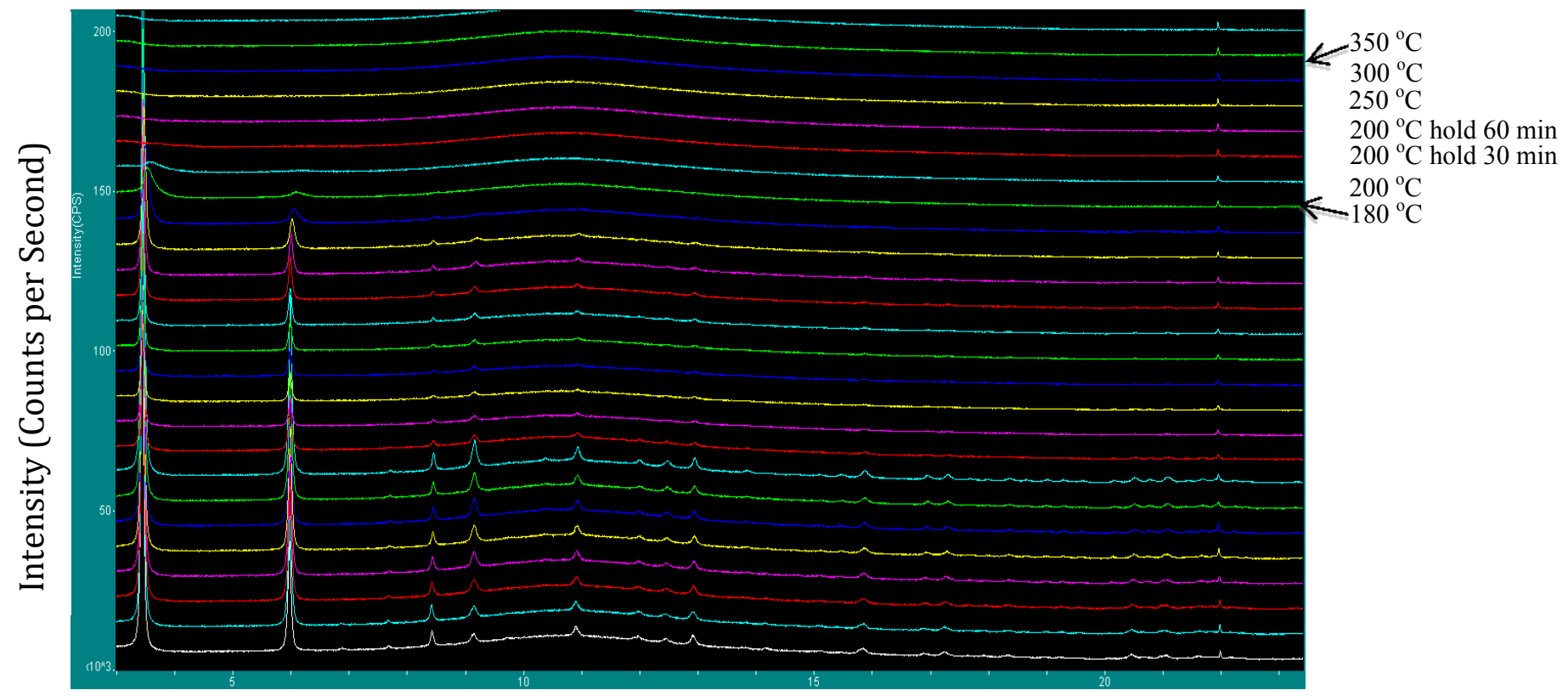

$2 \theta$

Figure 11. Ni/DOBDC activated at $200^{\circ} \mathrm{C}$ overnight.

The bottom spectrum in white was taken first $\left(35^{\circ} \mathrm{C}\right)$, the top spectrum in light blue was taken last and the spectra in between were taken consecutively, at approximately equal temperature intervals of $20^{\circ} \mathrm{C}$ up to the light blue line, above that the spectra are marked on the graph. These data show that sample quality deteriorated with time. 
Figure 12 shows the XRD spectra for the Ni/DOBDC after activation at $140^{\circ} \mathrm{C}$ and exposure to xenon. The peaks that change with time and temperature are at approximately 6.8 and $9.82 \Theta$ and are shown more clearly in the detail in (b). The appearance of peaks at 30 minutes and, to a greater extent, at 60 minutes indicates that the xenon may be interacting with the crystal structure of the MOF. After the sample is reheated to $140^{\circ} \mathrm{C}$ without additional xenon flow, small peaks are still observable at 6.8 and 9.8 .

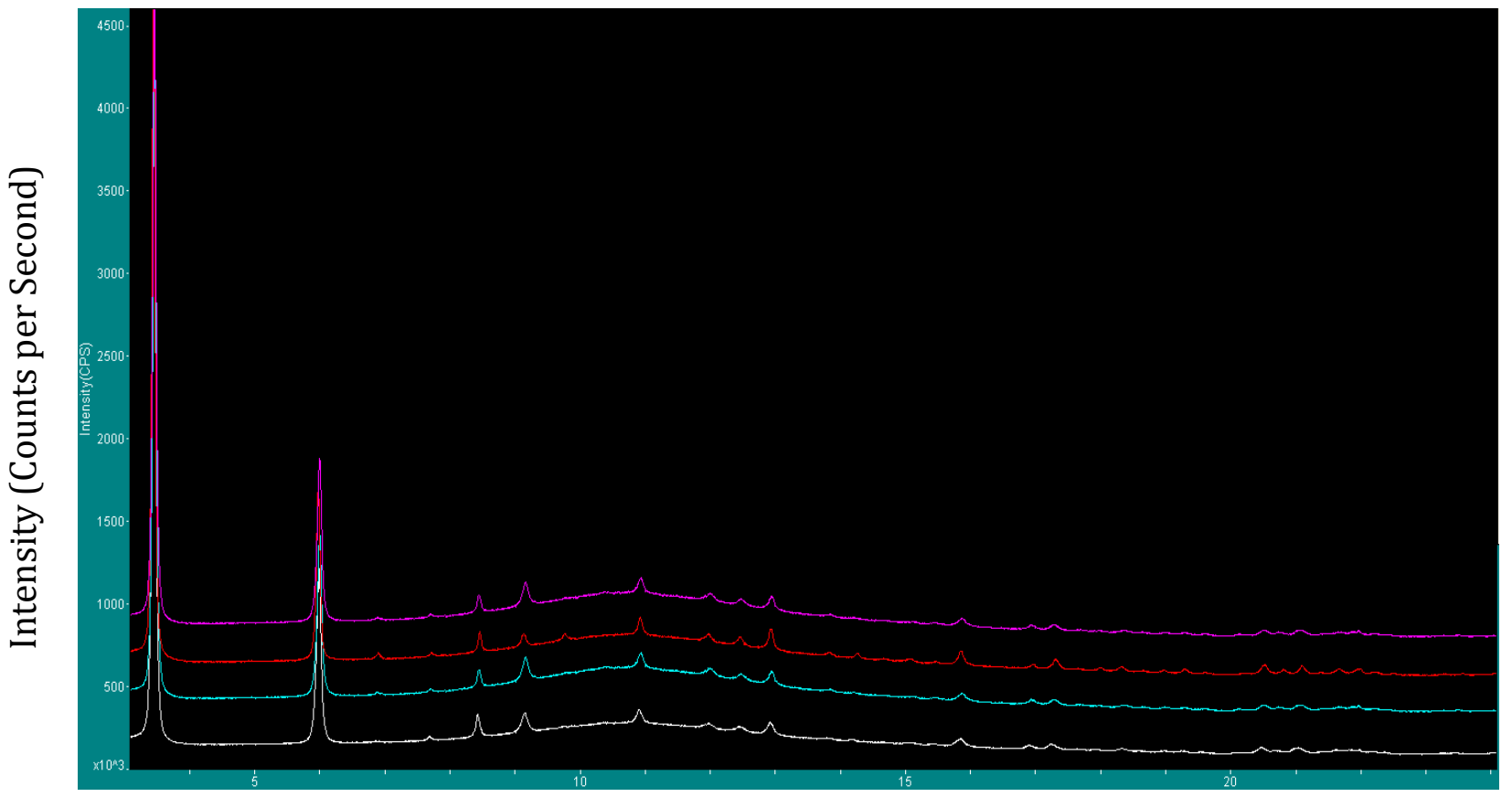

$2 \theta$

(a) XRD data for the $2 \theta$ range three to $\sim 25$ degrees.

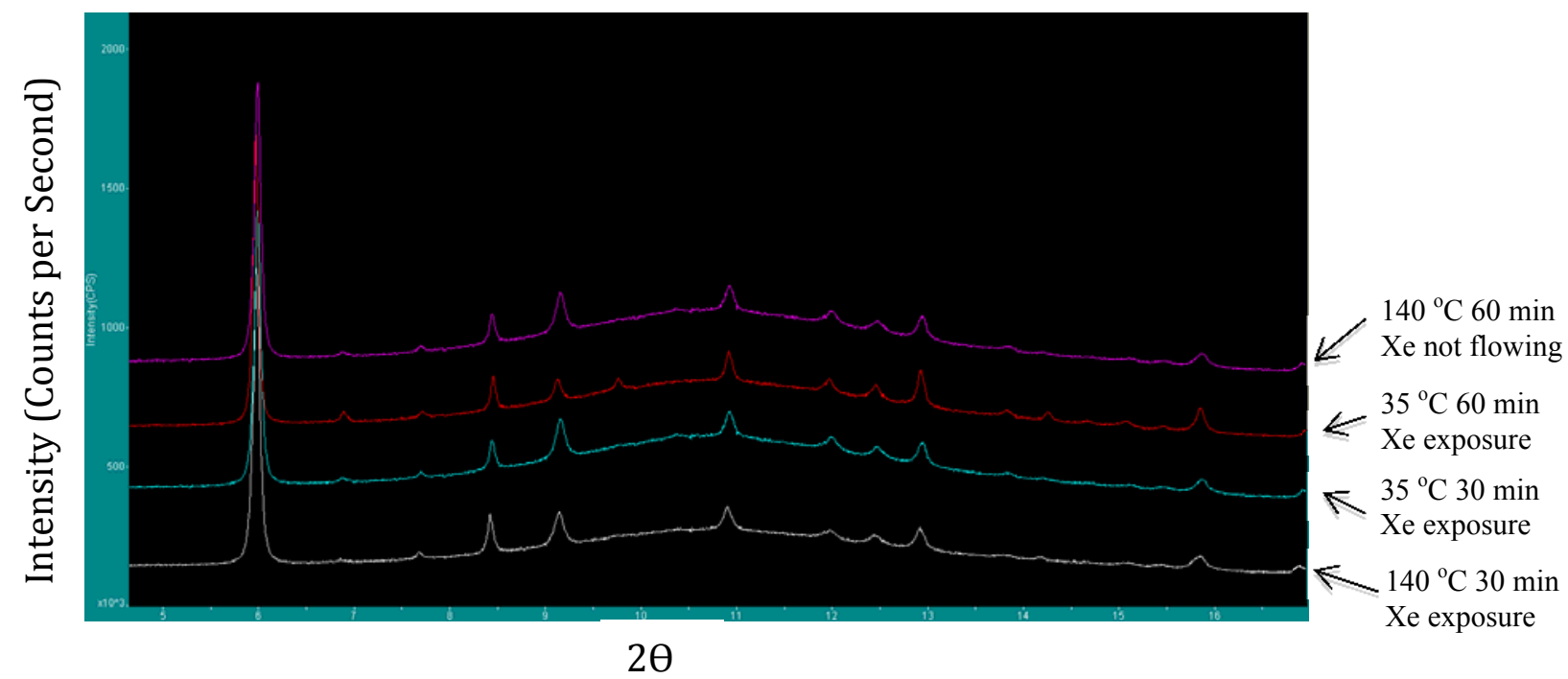

(b) Detail of (a) showing the $2 \theta$ range between 6 and 16 degrees.

Figure 12. XRD data for the Ni/DOBDC MOF after activation and exposure to xenon gas. Note the changes to the peaks at 6.8 and $9.82 \theta$. 
Figure 13 shows the effect of increasing temperature on Ni/DOBDC in the presence of xenon. Peaks at approximately 6.8 and $9.82 \theta$ indicate that xenon interacts with the structure of the MOF and that this interaction can be seen up to $180^{\circ} \mathrm{C}$ at which temperature the XRD spectrum deteriorates. This is an interesting result because the xenon does not appear to desorp from the MOF until higher temperatures. It conflicts with previous quantative gas adsorption measurements [14] but may be indicative of the structural interaction of the MOF with xenon. This result, combined with the result from Figure 12, may indicate that thermal vibrations hinder gas molecules from entering or exiting pores at higher temperatures. If a MOF could be developed that did not decompose at high temperature, this mechanism may make it possible to retain fission gases at reactor temperatures. However, more experimental information is needed to definitively draw this conclusion.

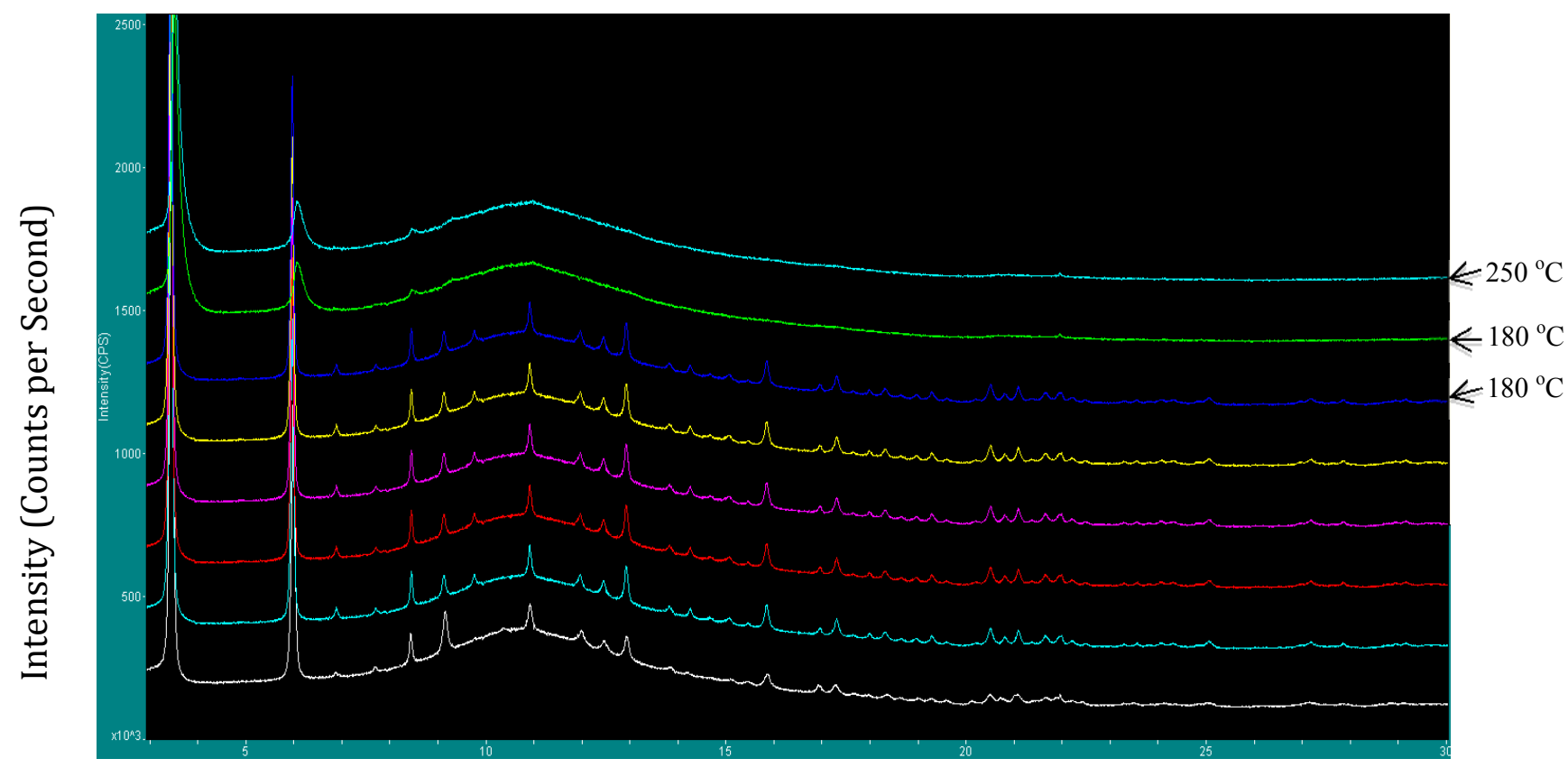

Figure 13. XRD data for Ni/DOBDC MOF after activation and exposure to xenon starting at $35^{\circ} \mathrm{C}$ for 60 minutes and increasing in temperature to $180^{\circ} \mathrm{C}$ in approximately equal temperature intervals.

The smooth spectra are as labeled on the graph. Note the changes in the peaks at 6.8 and $9.82 \Theta$ with time and temperature. 
The structure of the Ni/DOBDC MOF during interactions with krypton is shown in Figure 14. The appearance of the peaks at 6.8 and $9.82 \theta$ for the pink and red spectra indicate that there may by some change in the structure of the MOF due to the interaction with krypton. The peak shifts that can be observed at 8.5 and $92 \theta$ may also be due to structural changes in the material. Red and pink spectra are almost identical and indicate no change in the structure of the MOF.

Figure 15 shows the XRD spectra for the Basolite after activation at $140^{\circ} \mathrm{C}$ and exposure to xenon. The sharpness of the peaks indicate well developed crystalline structure consistent with the HRSEM micrograph in Figure 2. Based on the TGA results and the reported properties of Basolite, it is expected that xenon is adsorped on Basolite at low temperatures. However, this is not apparent from the data shown probably because the adsorption of xenon is not affecting the crytal structure of the Basolite. The disappearance of the peaks at $350^{\circ} \mathrm{C}$ indicates that the material became amorphous at this temperature.

The diffraction experiments at 16 and $80 \mathrm{keV}$ indicate that changes in the structure of $\mathrm{Ni} / \mathrm{DOBDC}$ with exposure to xenon and krypton can be observed with powder XRD. While the results are preliminary, deciphering the precise structural changes that are occurring is probably not feasible with powder XRD alone. Complimentary techniques, including PDF analysis, will be required and additional, better quality (less noisy) data needs to be obtained at high energies. 


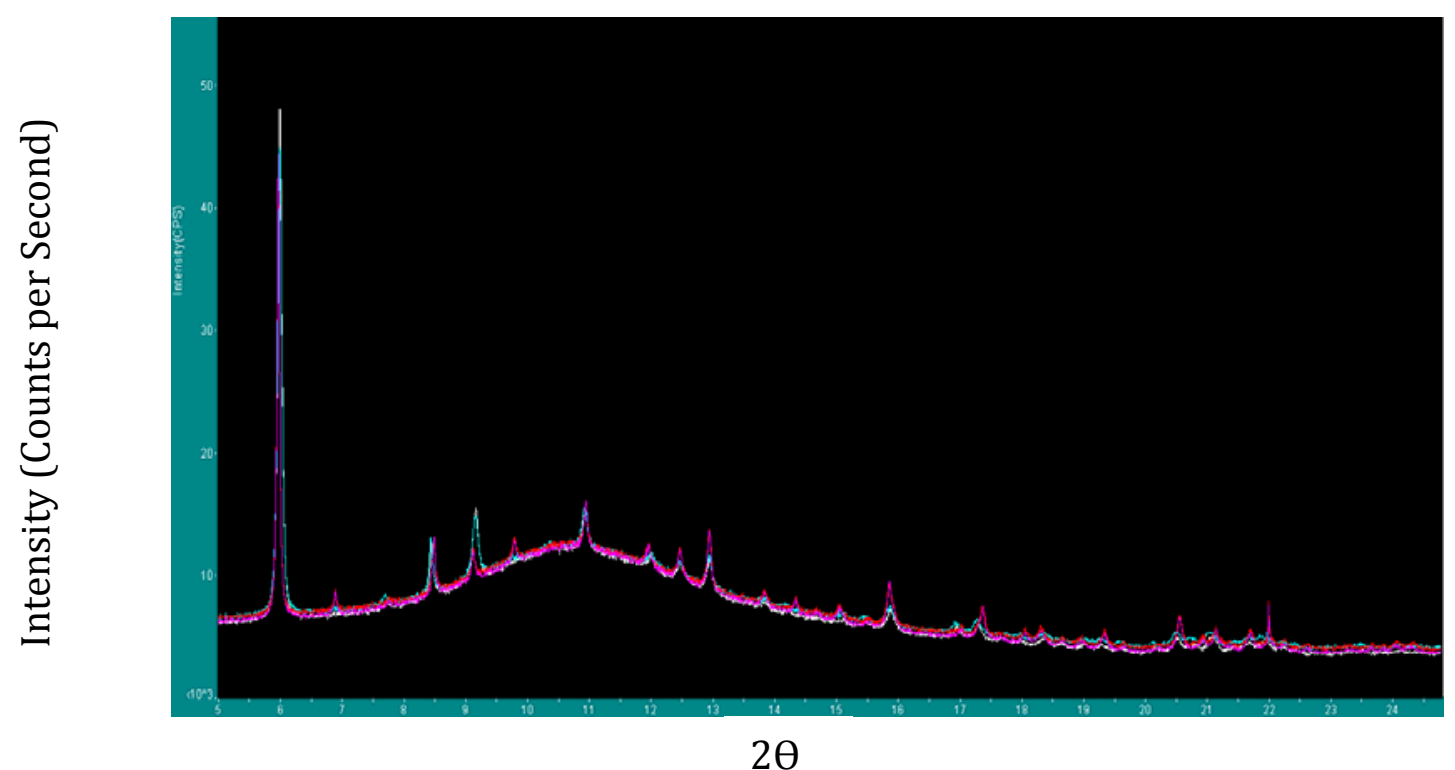

(a) XRD data for the $2 \theta$ range five to $\sim 25$ degrees

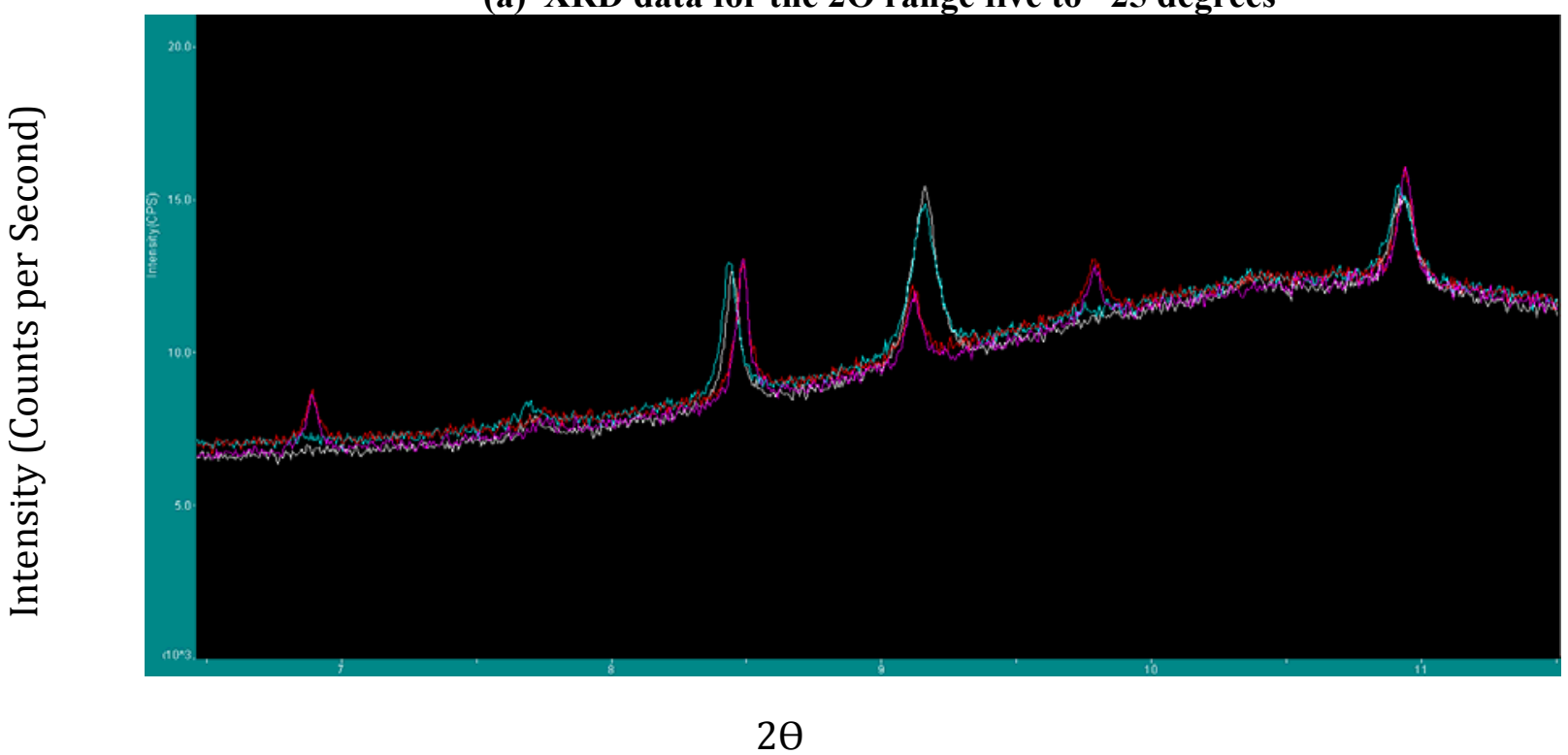

(b) Detail of (a) showing the $2 \theta$ range between 6 and 12 degrees

Figure 14. XRD data for Ni/DOBDC MOF after activation and exposure to krypton.

White spectrum is activated MOF at $140^{\circ} \mathrm{C}$ before krypton exposure, green spectrum is $140^{\circ} \mathrm{C}$ with 30 minute krypton exposure, red spectrum is $35^{\circ} \mathrm{C} 60$ minutes with flowing krypton and the pink is $35^{\circ} \mathrm{C} 60$ minutes without flowing krypton. 


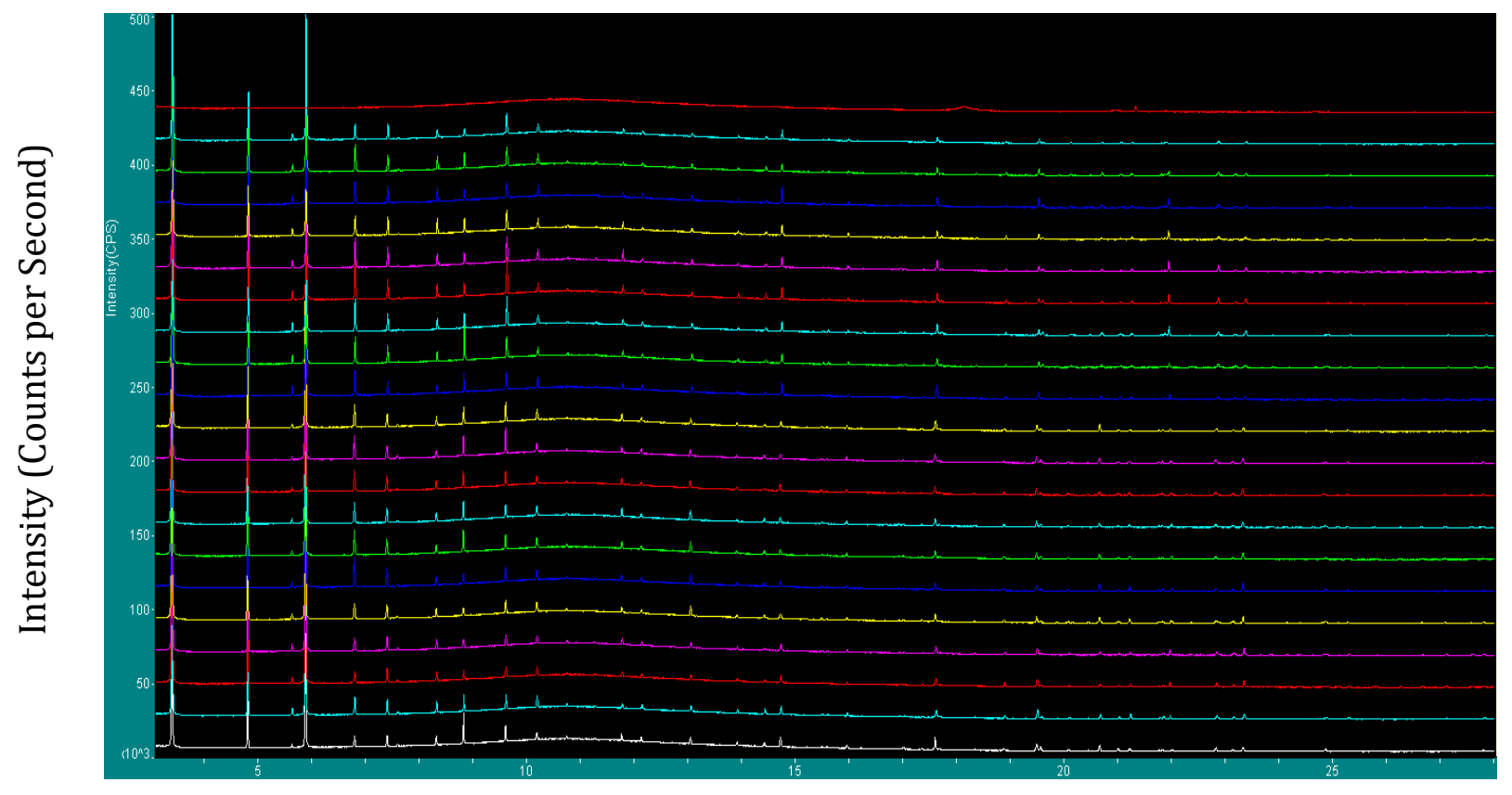

$2 \theta$

Figure 15. XRD data for Basolite showing scans from $35-350^{\circ} \mathrm{C}$ in flowing xenon.

The bottom spectrum in white was taken first $\left(35^{\circ} \mathrm{C}\right)$, the top spectrum in red was taken last $\left(350^{\circ} \mathrm{C}\right)$ and the spectra in between were taken consecutively, at approximately equal temperature intervals. The material did not show any structural changes due interaction with xenon. It decomposed at $350^{\circ} \mathrm{C}$. 


\section{CONCLUSIONS}

1. Lowering the fission gas pressure in the plenum is a challenging research goal, and it is unlikely that bulk materials can getter noble gases on an exposed surface at reactor temperatures. However, it may be possible to design a material for this application. Therefore, identifying materials that adsorb noble gases and understanding the nature of the interaction is an important first step. If such a material can be found, it may be possible to modify it for use at higher temperatures.

2. The TGA experiments performed at BNL are inconclusive. Additional confirmatory experiments may be recommended, but the experimental design and test protocol has not been determined.

3. New candidate materials will be considered at any time during the research to take advantage of recent advancements in material science.

4. Candidate materials should be evaluated using BET surface area measurements and gas adsorption measurements.

5. The in situ powder XRD measurements completed at the NSLS indicate that changes in the diffraction spectra of some MOF materials (Ni/DOBDC) can be observed during the interaction of the MOF with xenon and krypton.

6. More high energy diffraction data is needed to perform a PDF analysis and additional complimentary techniques may also be required to determine the precise structural interaction between the xenon and Ni/DOBDC.

7. A closer collaboration with PNNL will be pursued with a formal nondisclosure agreement. PNNL has extensive experience and knowledge of MOFs that can greatly benefit the research. 


\section{REFERENCES}

1. Parker, Sybil P., "McGraw-Hill Encyclopedia of Chemistry," Second Edition, McGrawHill, Inc.

2. Keller, Jürgen U. and Staudt, Reiner, "Gas Adsorption Equilibria: Experimental Methods and Adsorptive Isotherms," Universität Siegen, Germany, Springer, 2005.

3. Redhead, P. A., et al., Physical Basis of Ultrahigh Vacuum, p. 28, American Institute of Physics, London, 1993.

4. Email communication between Patrick Looney and William Horak, May 24, 2012.

5. Project Review Meeting at Brookhaven National Laboratory between Patrick Looney, Jason Graetz, John Valente and Lynne Ecker, September 12, 2012.

6. Project Review Meeting at Brookhaven National Laboratory with Peter Johnson and Lynne Ecker, October 4, 2012.

7. Meek, Scott T., et al., "Effects of Polarizability on the Adsorption of Noble Gases at Low Pressures in Monohalogenated Isoreticular Metal-Organic Frameworks," J. of Physical Chemistry, p. 19765-19772, August 2012.

8. Greathouse, Jeffrey A., et al., "Adsorption and Separation of Noble Gases by IRMOF-1: Grand Canonical Monte Carlo Simulations,” Ind. Eng. Chem. Res., 48, p. 3425-3421, 2009.

9. Thallapally, Praveen K., Grate, Jay W., and Motkuri, Radha Kishan, "Facile Xenon Capture and Release at Room Temperature Using a Metal-Organic Framework: A Comparison with Activated Charcoal," Chem Commun., 48, p. 347-349, 2012.

10. Fernandez, Carlos A., et al., "Switching Kr/Xe Selectivity with Temperature in a MetalOrganic Framework,” J. Am. Chem. Soc., 134, p. 9046-9049, 2012.

11. Zamaro, Juan M., et al., "HKUST-1 MOF: A Matrix to Synthesize $\mathrm{CuO}$ and $\mathrm{CuO}-\mathrm{CeO}_{2}$ Nanoparticle Catalysts for CO Oxidation," Chem. Eng. Journal, 195-196, p. 180-187, 2012.

12. Nobar, S. Najafi, and Farooq, S., "Experimental and Modeling Study of Adsorption and Diffusion of Gases in Cu-BTC," Chem. Eng. Sci., May 2012.

13. Liu, Jian, et al., "Selective $\mathrm{CO}_{2}$ Capture from Flue Gas Using Metal-Organic Frameworks-A Fixed Bed Study," J. Phys. Chem., 116, p. 9575-9581, 2012.

14. Liu, Jian, Thallapally, Praveen, K., Strachan, Denis, M., "Metal-Organic Frameworks for Removal of Xe and Kr from Nuclear Fuel Reprocessing Plant," Langmuir, July 2012. 
15. Aoshima, Masayuki, Fukasawa, Kiyotake, and Kaneko, Katsumi, "Micropore Filling of Supercritical Xe in Micropores of Activated Carbon Fibers," J. of Colloid and Interface Sci., 222, p. 179-183, 2000.

16. Wandelt, K., and Hulse, J. E., "Xenon Adsorption on Palladium. I. The Homogeneous (110), (100) and (111) Surfaces,” J. Chem. Phys., 80(3), p. 1340-1352, February 1984.

17. Da Silva, Juarez, L. F., and Stampfl, Catherine, "Trends in Adsorption of Noble Gases $\mathrm{He}, \mathrm{Ne}, \mathrm{Ar}, \mathrm{Kr}$, and $\mathrm{Xe}$ on $\mathrm{Pd}(111)(\sqrt{3} x \sqrt{3}) \mathrm{R} 30^{\circ}$ : All-Electron Density-Functional Calculations," Physical Review, 77, p. 45401, 2008.

18. Seyller, Th., et al., "Observation of Top-Site Adsorption for Xe on $\mathrm{Cu}(111)$," Chemical Physics Letters, 291, p. 567-572, 1998.

19. Sun, X., and Yamauchi, Y., "First-Principles Calculations of Xe-adsorbed Pd(111) and $\mathrm{Cu}(111)$ Surfaces with an Empirical Correction of van der Waals Interactions," J. of Applied Physics, 110, p. 103701, 2011.

20. Sozzani, Piero, et al., "Nanoporosity of an Organo-Clay Shown by Hyperpolarized Xenon and 2D NMR Spectroscopy," Chem. Commun., p. 1921-1923, 2006.

21. Kuznetsova, A., and Yates, Jr., J.T., "Physical Adsorption of Xenon in Open Single Walled Carbon Nanotubes: Observation of a Quasi-One-Dimensional Confined Xe Phase," J. Chem. Physics, 112:21, p. 9590-9598, 2000.

22. Foroutan, Masumeh, and Nasrabadi, Amir Taghavi, "Adsorption and Separation of Binary Mixtures of Noble Gases on Single-walled Carbon Nanotube Bundles," Physica E., 43, p. 851-856, 2011.

23. Saxton, Carl, G., et al., "Xenon Adsorption in Synthetic Chabazite Zeolites," Microporous and Mesoporous Materials, 129, p. 68-73, 2010.

24. Yumura, Takashi, et al., "Site-Specific Xe Additions into Cu-ZSM-5 Zeolite," Physical Chemistry Chemical Physics, 12, p. 2392-2400, 2010.

25. Farrusseng, David, et al., "Heats of Adsorption for Seven Gases in Three Metal-Organic Frameworks: Systematic Comparison of Experiment and Simulation," Langmuir, 25(13), p. 7383-7388, 2009.

26. Sigma Aldrich Website. http://www.sigmaaldrich.com/analyticalchromatography/analytical-products.html?TablePage $=104061281$ 


\section{APPENDIX 1. EXPERIMENTAL TECHNIQUES}

\section{Brunauer-Emmett-Teller (BET) Measurements:}

Candidate getter materials may be characterized for their adsorption and inherent porosity by BET measurements. These studies will be done in two steps:

Step 1. Qualify the candidates on porosity: Measure the basic properties of the materials, such as surface area and pore volume, which are crucial to assess the gas adsorption capability of the materials. Surface area of the samples may be measured on an Autosorb-1-MP automatic surface area and pore size analyzer (Quantachrome Instrument Corp.) by nitrogen adsorption/desorption techniques. Prior to surface area measurements, the surface of samples will be cleaned of contaminants by degassing the sample. Degassing will be done using the built-in vacuum system of the NOVA series, and sample will be heated at $60^{\circ} \mathrm{C}$ for several hours (24-48 hours). Figure A1 depicts how a solid particle with pores may look after the degassing pretreatment.
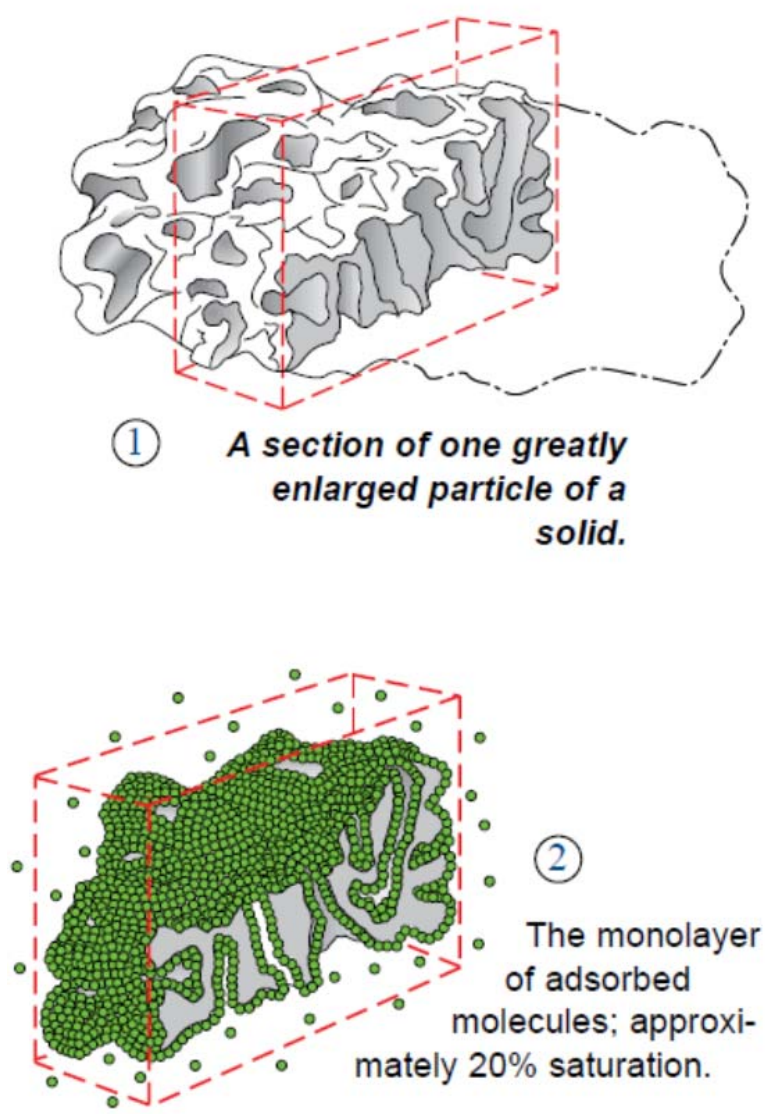

Figure A1. Schematic showing the solid sample surface during 1- degassing of the sample, 2- monolayer formation. (Schematic from Quantachrome Instruments [A1]).

The surface area can be calculated by the BET method from the adsorption isotherm at $77 \mathrm{~K}$. After degassing, the sample is cooled by an external bath and small amounts of adsorbate (nitrogen gas) are introduced in the sample chamber under vacuum. The adsorbate gas molecules adsorb on the surface of the solid sample (the adsorbent) forming a thin layer that covers the 
whole adsorbent surface. Using BET theory, the number of molecules required to form a complete monolayer (Figure A1.2) on the surface can be estimated. From this measurement and the cross-sectional area of the adsorbate molecule, the surface area of the solid sample can be assessed. After initial testing, the pore size distribution may also be determined [A1].

Step 2. Noble gas adsorption studies under high T and P: Gas adsorption measurements may be performed using an automated gas sorption analyzer (Autosorb-1-MP) from Quantachrome, Inc. Prior to measurements, the sample surface will be cleaned to desorb contaminants and some of the samples (e.g., Basolite) may require an additional activation step. Before introducing noble gas (krypton or xenon) for gas adsorption measurements on the sample, the system will be purged with five gas cycles (introduction and evacuation), and the sample chamber will be pressurized at fixed initial pressure and left to equilibrate until no further pressure variation is detected. Then gas will be added to increase the pressure. The sample will be maintained in this condition, and the pressure drop due to adsorption will be measured. The pressure drop will then be correlated directly to amount of gas adsorbed. Adsorption analysis will be done at various pressures between 0.1 and 1 bar. The volume adsorbed per unit mass of material will be a function of pressure for each temperature. In addition, gas adsorption measurements will be done at various temperatures including $25^{\circ} \mathrm{C}$ up to $300^{\circ} \mathrm{C}$. Such noble gas adsorption studies at high $\mathrm{T}$ and $\mathrm{P}$ will help us in comparing the capability of candidates in gettering noble gases for nuclear applications. For high $\mathrm{P}$ (above 1 bar) measurements, the Quadrasorb-SI from Quantachrome may be used [A1].

\section{Surface Sensitive Characterization Techniques for Xenon}

Low Energy Election Diffraction (LEED) is a highly surface sensitive technique and is commonly used to determine the surface structure of crystalline materials. It has been used to determine the ordering, registry and sensitivity to impurities of xenon layers on surfaces [A2]. Other techniques that are available at the synchrotron for studying adsorption are grazing incidence X-ray diffraction (GIXD), X-ray reflectivity, and grazing incidence small angle X-ray scattering (GISAXS) [A3]. GIXD has been used to study the out of plane structure of xenon layers adsorbed to the silver (111) surface [A4]. In addition, the photoemission of adsorbed xenon (PAX) can be used to probe the local work function of real surfaces and is sensitive to the crystal structure and structural and chemical imperfections [A5]. 


\section{References}

A1. Quantochrome Instruments, Inc. website: http://www.quantachrome.com/

A2. Shin, Heekeun, et al., "Ordering and Growth of Xe Films on the 10-fold Quasicrystalline Approximant $\mathrm{Al}_{13} \mathrm{Co}_{4}(100)$ Surface," Physical Review B, 84, p. 115454, 2011.

A3. Arnold, Thomas, and Clarke, Stuart, M., "Diffraction from Physisorbed Layers," Current Opinion in Colloid and Interface Science, 17, p. 23-32, 2012.

A4. Dai, Pengcheng, et al., "Synchrotron X-Ray Diffraction Study of the Structure and Growth of Xe Films Adsorbed on the Ag(111) Surface," Physical Review B, 59:23, p. 15 464-15 478, 1999.

A5. Wandelt, K., "The Local Work Function: Concept and Implications," Applied Surface Science, 111, p. 1-10, 1997. 